\title{
Chemical Modification of Chitosan in order to Inverse Swelling Behavior: Comparison between Cross-linking, Michael Addition and Graft Copolymerization Reactions
}

\author{
Solmaz Zakhireh ${ }^{1}$, Mehrdad Mahkam², Yousef Toomari ${ }^{3}$ and Saeed Jafarirad $^{4}$ \\ ${ }^{1}$ Department of Chemistry, Tabriz Branch, Islamic Azad University, Tabriz, Iran \\ ${ }^{2}$ Chemistry Department, Faculty of Science, Azarbaijan Shahid Madani University, Tabriz, Iran \\ ${ }^{3}$ Laboratory of Dendrimers and Nanobiopolymers, Faculty of Chemistry, University of Tabriz, Tabriz, Iran \\ ${ }^{4}$ Research Institute for Fundamental Sciences (RIFS), University of Tabriz, Tabriz, Iran
}

Received: April 10, 2015; Accepted: June 01, 2015; Published (Web): July 21, 2015

\begin{abstract}
In the present work, three methods were used to inverse chitosan swelling behavior including, conjugation of p-amino benzoic acid (PABA) on cross-linked chitosan in different condition, Michael addition reaction of acrylic acid (AA) on chitosan and finally graft copolymerization of methacrylic acid (MMA) on chitosan. We couldn't inverse chitosan swelling behavior by the Michael addition reaction of acrylic acid, but conjugation of PABA on cross-linked chitosan inversed the chitosan swelling behavior. In addition, the graft copolymerization of MMA on chitosan was also successful. In general, the third method, among the above-mentioned modifications provided the best results.
\end{abstract}

Key words: Chitosan; Cross-linking; Michael addition; Graft copolymerization; Swelling.

\section{Introduction}

Chitosan, the cationic polysaccharide derived from chitin, is a biodegradable (Park et al., 2003), biocompatible, non-toxic, antimicrobial and renewable biopolymer (Meng et al., 2009; Fang et al., 2010; Jayakumar et al., 2010; Benhabiles et al., 2012; SaberSamandari et al., 2012; Saita et al., 2012). It has found applications in fields, for instance, medicine, cologne, cosmetics, food industry and agriculture (Gerasimenko et al., 2004; Lin et al., 2014; Ai et al., 2012; Domingues et al., 2012). Chitosan, because of the presence of a primary amino group in most of the sugar units, dissolves in dilute acids, but is insoluble in organic solvents, water and alkaline solutions (Goosen, 1996). Most of the real-life applications for any chemical substance, whether natural or synthetic, require the chemicals to be processable. In this regard, chitosan has a great drawback because of the solubility problems in neutral and basic aqueous media as well as commonly used organic solvents. The pKa value of the primary amino groups in chitosan is determined to be around 6.5 (Strand et al., 2001). Even though chitosan and its derivatives are soluble at $\mathrm{pH}$ values of lower than 6
(Wang and Wang, 2011), many of its applications in the neutral or basic media, including those of physiological behaviors, may not be recognized. Therefore, they will trigger an immediate precipitation for the $\mathrm{pH}$ under such condition. On the other hand, acidic solutions, in which chitosan is fairly soluble, may not be desirable in many of its applications, especially those in medicines, cosmetics, and foods. There have been several approaches towards improving the solubility of chitosan at neutral $\mathrm{pH}$ such as strongly hydrophilic substituent addition, ionic derivatization, for example quaternary amination, carboxymethylation and sulfatation (Baumann and Faust, 2001). In addition to the ionic derivatives, a few non-ionic polar derivatives of chitosan have been prepared, for example, graft of polyacrylic acid and various polar polyacrylates.

In this study, briefly, we used three methods to inverse chitosan swelling behavior. Our goal from inversing swelling behavior is to reach the most swelling at $\mathrm{pH} 7.4$ instead of $\mathrm{pH}$ 1. Therefore, three chemical modifications were applied including: i) conjugation of p-amino benzoic acid on cross-linked chitosan in different conditions that

Correspondence to: S. Jafarirad, E-mail: jafarirad@tabrizu.ac.ir and M. Mahkam, E-mail: mmahkam@yahoo.com 
can't inverse the swelling behavior because of having carboxylic group, but also have pharmaceutical properties such as UV filtration, ii) Michael addition reaction of acrylic acid (AA) on chitosan, and finally, iii) graft copolymerization of methacrylic acid (MMA) on chitosan.

\section{Materials and Methods}

Materials: The chitosan sample $(75-85 \%$ the degree of deacetylation) and glutaraldehyde were purchased from Sigma-Aldrich Chemical Company, USA. The solvents and reagents were obtained from Fluka. FT-IR spectra were measured on a FT-IR Bruker PS 15 spectrophotometer.

Chitosan conductometric titration: Chitosan (0.1g) was dissolved in $20 \mathrm{ml}$ aqueous hydrochloric acid $(0.1 \mathrm{M})$ and was titrated by aqueous $\mathrm{NaOH}(0.1 \quad M)$ and investigated by conductometric system.

Preparation of cross-linked chitosan films using glutaraldehyde with different concentrations 6\% (w/w) (FG6) and 18\% (w/w) (FG18): Chitosan (1 g) was dissolved in $100 \mathrm{ml}$ of acetic acid solution (1.5 vol. \%), filtered and poured onto a glass plate, then dried at $60^{\circ} \mathrm{C}$ in an oven for $3 \mathrm{~h}$. Aqueous solution of $\mathrm{NaOH}(2.0 \% \mathrm{wt}$.) was added to the glass plate in order to neutralize the acetic acid in the chitosan film. The films were repeatedly washed with deionized water and finally dried again. These films were subsequently kept in $50 \mathrm{~mL}$ neutral solution of glutaraldehyde with different concentrations $6 \%(\mathrm{w} / \mathrm{w})$ and $18 \%(\mathrm{w} / \mathrm{w})$ at room temperature. After $15 \mathrm{~h}$, the films were separated and washed extensively with a $0.2 \mathrm{M} \mathrm{NaCl}$ solution to remove the free glutaraldehyde.

Synthesis of PABA-conjugated chitosan films (FG6PABA) and (FG18-PABA-1) in Organic Solvent: The synthetic procedure for both films was same, for example about $\mathrm{FG}_{6}-\mathrm{PABA}$, cross-linked chitosan film $\mathrm{FG}_{6}(0.2 \mathrm{~g})$ and p-amino benzoic acid $(0.5 \mathrm{~g}, 3.5 \mathrm{mmol})$ were refluxed in $6 \mathrm{~mL}$ methanol and $0.6 \mathrm{~mL}$ acetic acid overnight. After completion of Schiff-base reaction, the product was washed with methanol and acetone and dried in vacuum.

Synthesis of PABA-conjugated chitosan films in aqueous solvent at room temperature (FG18-PABA-2) and $60^{\circ} \mathrm{C}$ (FG18-PABA-3): For the preparation of $\mathrm{FG}_{18^{-}}$ PABA-2, $\mathrm{FG}_{18}(0.2 \mathrm{~g})$ was added to $\mathrm{p}$-amino benzoic acid $(0.5 \mathrm{~g}, 3.5 \mathrm{mmol})$ aqueous solution and the mixture was kept for $24 \mathrm{~h}$ at room temperature ( $\mathrm{rt})$. To prepare of $\mathrm{FG}_{18^{-}}$ PABA-3, the above-mentioned procedure was used except that the mixture was finally stirred slowly and heated up to $60^{\circ} \mathrm{C}$ for $1 \mathrm{~h}$.

Synthesis of N-carboxy ethyl chitosan; CECTS-1 (1:10 mol$/ \mathrm{mol})$ and CECTS-2 (1:50 mol$/ \mathrm{mol}):$ CECTS-1 and CECTS-2 were prepared through Michael addition reaction of acrylic acid (AA) on chitosan according published method (Aoi et al., 2000) with some modifications. Briefly, for preparation of CECTS-2, chitosan (1g, $-\mathrm{NH}_{2}$ ca. $7 \mathrm{mmol}$ ) was dissolved in $20 \mathrm{~g}$ of aqueous solution containing $25.22 \mathrm{~g}$ ( $350 \mathrm{mmol})$ of AA. Stirring gave a transparent and viscous solution, which was stirred at $50^{\circ} \mathrm{C}$ for $12 \mathrm{~h}$. The raw product was precipitated by the addition of excess acetone after the completion of resulting the reaction. For complete purification, the product was dissolved in distilled water and left for 2 days. Eventually, the product was reprecipitated in acetone and then dried under vacuum to generate the purified product.

Graft copolymerization of methacrylic acid with CECTS-2 (CECTS-2-g-PMMA): Graft copolymer CECTS2-g-PMMA was homogeneously synthesized in aqueous solution. CECTS-2 (1g) was dissolved in $50 \mathrm{ml}$ distilled water. Heating and stirring gave a viscous solution. Then CECTS-2 was graft copolymerized with methacrylic acid ( $3 \mathrm{~g}, 35 \mathrm{mmol}$ ) by using the $\mathrm{N}, \mathrm{N}^{\prime}$-methylenebisacrylamide as a cross-linking agent $(0.05 \mathrm{~g}, 0.32 \mathrm{mmol})$ and potassium persulfate as an initiator $(0.1 \mathrm{~g}, 0.37 \mathrm{mmol})$ at $80^{\circ} \mathrm{C}$. After the specific time $(10 \mathrm{~h})$ the precipitated network polymer was collected and after washing with ethanol dried under vacuum.

Measurement of swelling ratio (SW \%): $20 \mathrm{mg}$ of dry polymers were immersed in various buffer solutions $(\mathrm{pH}$ 7.4 and $\mathrm{pH} 1$ ) at $37^{\circ} \mathrm{C}$. The weight of the swollen samples was measured for $4 \mathrm{~h}$ with one hour intervals. The procedure was repeated until there was no further increase of weight. The SW (\%) was calculated according to equation show below:

$$
\mathrm{SW}(\%)=\left[\left(\mathrm{W}_{2}-\mathrm{W}_{1}\right) / \mathrm{W}_{1}\right] \times 100
$$

Where $\mathrm{W}_{1}$ is the initial weight of sample and $\mathrm{W}_{2}$ is the weight of sample after swelling in buffer solution for $4 \mathrm{~h}$. 


\section{Results and Discussion}

Conductometric titration of chitosan: Conductometric titration curve of a chitosan solution in aqueous hydrochloric acid is shown in figure 1. As shown in figure 1 , the electrical conductivity of initial section in the titration curve decreased due to neutralization of the excess hydrochloric acid with aqueous sodium hydroxide and then it increased due to displacement of $\mathrm{HCl}$ bound to the primary amino groups of chitosan hydrochloride. The addition of excess aqueous alkali strongly increased the electrical conductivity. The resulting titration curve has two breaks corresponding to the final titration points
(FTPs) of free and bound $\mathrm{HCl}$, respectively. The correct positions of these points can be determined by linear extra polation of the adjacent portions of the titration curve. The chitosan concentration in the solution was determined from the difference between aqueous alkali volumes corresponding to the second and first FTPs, respectively.

Study of cross-linking condition, solvent and temperature effects on FG6-PABA, FG18-PABA- $n$; ( $n=1$ $3)$ : As could be seen in figure 2, glutaraldehyde crosslinked chitosan films of $\mathrm{FG}_{6}$ and $\mathrm{FG}_{18}$ reacted with $\mathrm{p}$ amino benzoic acid for the inversion of chitosan swelling.

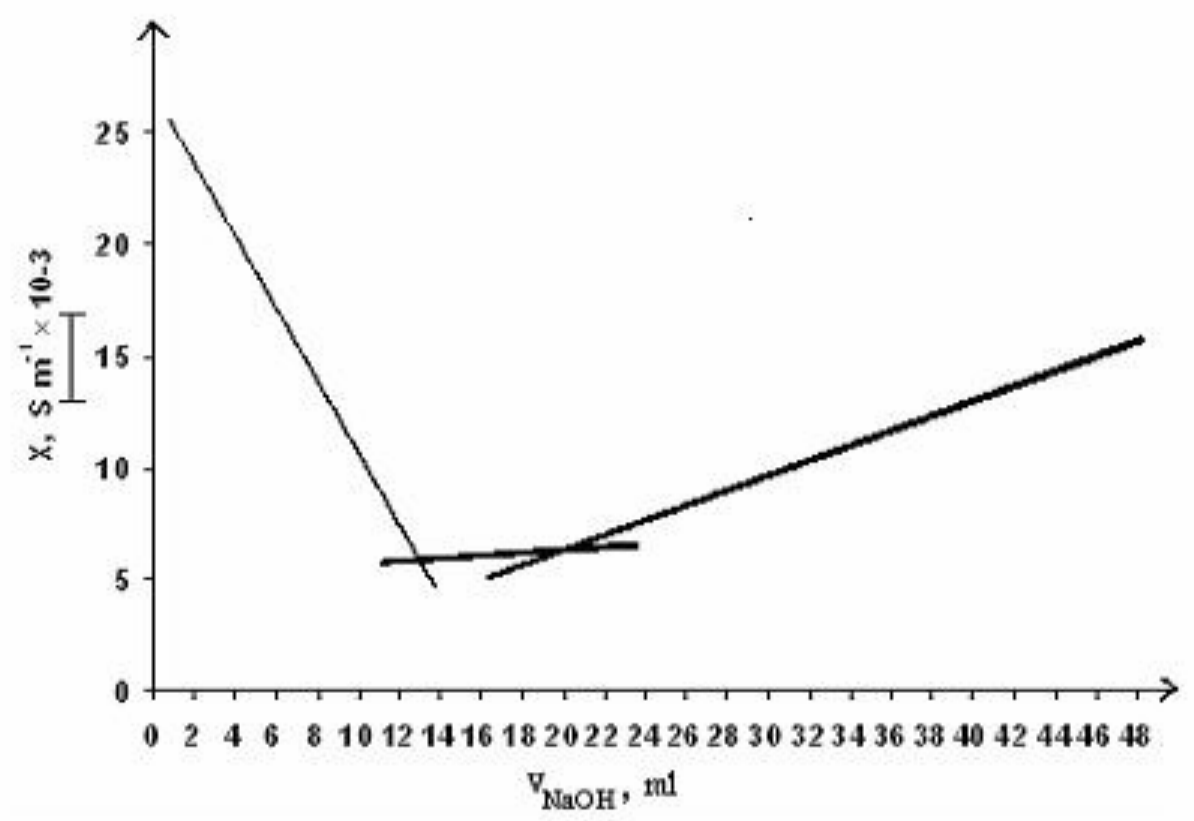

Figure 1. Conductometric titration curves of chitosan in hydrochloric acid solution.

The reactive amino groups of chitosan films were participated in a Schiff-base reaction with glutaraldehyde that had at least two reactive functional groups. Some of these functional groups in the other side of the glutaraldehyde chain remain free to react with drug. Preliminary trials with $6 \%$ and $18 \%$ of glutaraldehyde were carried out to evaluate the effects of cross-linker on chitosan swelling. The experimental data have obviously showed that the degree of cross-linking has considerably influenced the conjugation of PABA on the cross linkedchitosan films. Based on our results, higher concentrations (18\%) of glutaraldehyde showed high conjugation efficiency in film. At the next step, the effect of solvent change on conjugation has been investigated. Based on
FT-IR spectra (Figure 3C and E) as well as swelling behavior (Figure 6B and D), the amount of carboxylic acid groups from PABA in water are more than the same groups in methanol solvent. Therefore, it implies the high conjugation in water relative to methanol. In addition, as it has been known the green aspects of water relative to any other organic solvents made it the ideal candid for all chemical optimizations. Moreover, we studied the yield of PABA conjugation on cross-linked polymers at two temperatures $\left(\mathrm{T}=\right.$ room temperature and $\left.60^{\circ} \mathrm{C}\right)$. Based on FT-IR spectra (Figure 3D and E) as well as swelling behavior (Figure 6C and D) the amount of PABA conjugation at $60{ }^{\circ} \mathrm{C}$ is more than that of at room temperature. 


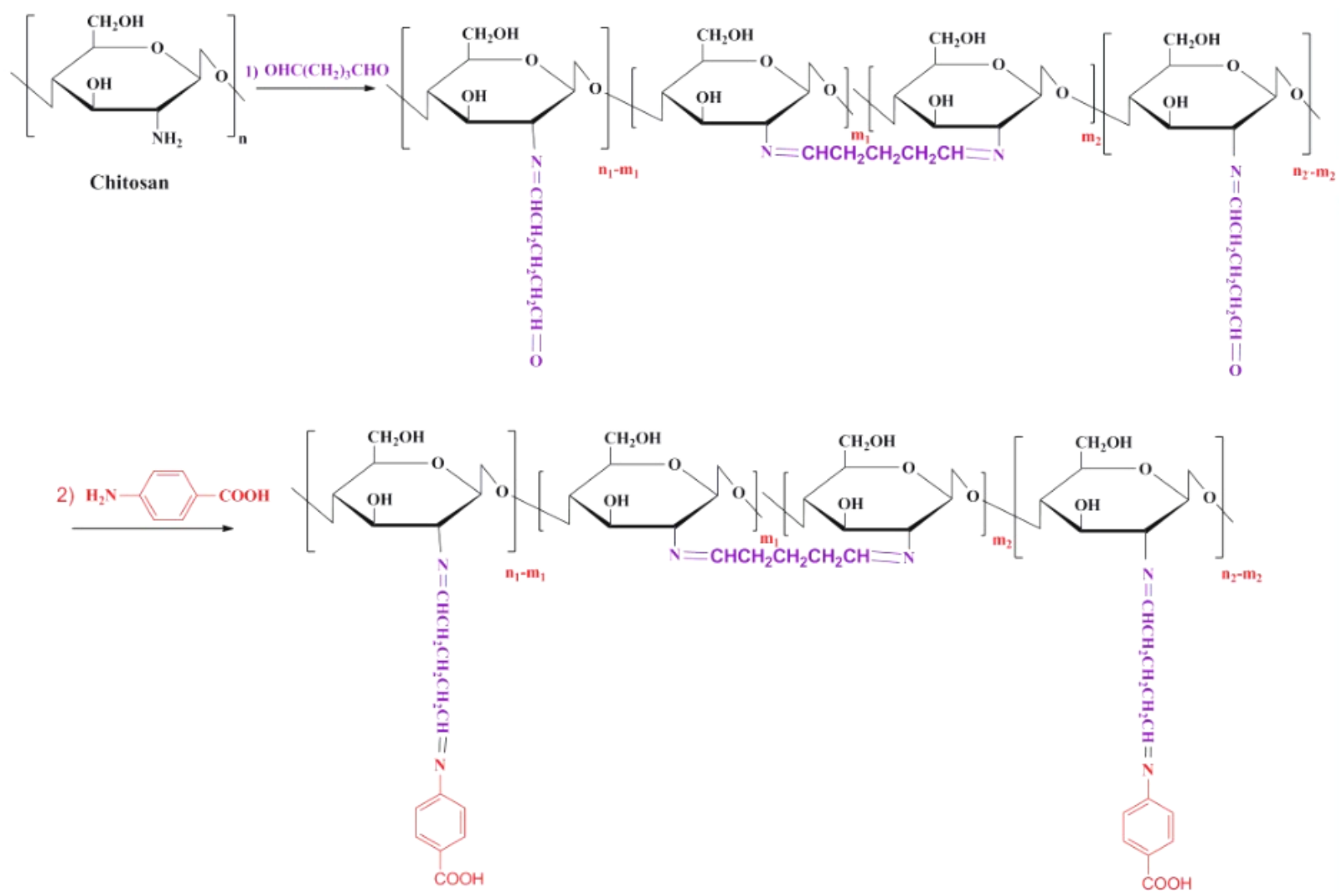

Figure 2. Synthesis of PABA-conjugated chitosan films.

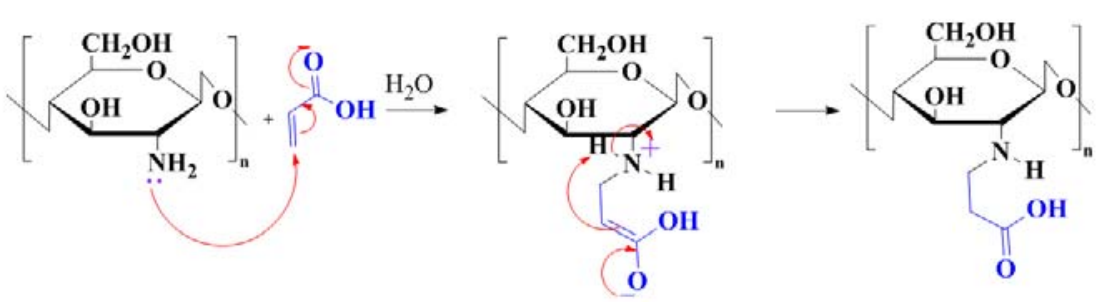

Figure 3. FT-IR spectra of A: Chitosan, B: FG6-PABA, C: FG18-PABA-1, D: FG18-PABA-2,E: FG18-PABA-3, F: CECTS-1, G: CECTS-2 and H: CECTS-2-g-PMMA.

Characterization of FG6-PABA, FG18-PABA-n; ( $n=1-3)$ : The FT-IR spectra of drug conjugated films, for instance $\mathrm{FG}_{18}$-PABA-1, represent typical absorption band at $3448 \mathrm{~cm}^{-1}$ which shows the presence of the respective absorption of hydroxyl, amine and acid groups (Figure 3C). Furthermore, $\mathrm{C}-\mathrm{H}$ asymmetrical stretching, $\mathrm{C}-\mathrm{H}$ bending, $\mathrm{N}-\mathrm{H}$ bending and $\mathrm{C}=\mathrm{C}$ stretching vibrations could be seen at 2854-2925, 1459, 1560 and $1637 \mathrm{~cm}^{-1}$, respectively. In addition, the respective peaks at 1654 , 1719 and $1735 \mathrm{~cm}^{-1}$ could be assigned to the stretching vibration of carbonyl groups of amide (respective to acetylated amine groups), carboxylic acid and free aldehyde groups. The C-N linkage has the characteristic peak near $1261 \mathrm{~cm}^{-1}$, while the peaks of $1648,1686 \mathrm{~cm}^{-1}$ stand for the stretching vibrations of $\mathrm{C}=\mathrm{N}$ in Schiff-base produced by the reaction of glutaraldehyde: p-amino benzoic acid and glutaraldehyde: chitosan respectively. Based on Figure 3B-E, in respect of the peak's width of carboxylic acid $\left(>3000 \mathrm{~cm}^{-1}\right)$, the amounts of carboxylic acid in $\mathrm{FG}_{6}$-PABA (Fig 3B) and $\mathrm{FG}_{18}-\mathrm{PABA}-2$ (Fig 3 D), 
are less than $\mathrm{FG}_{18}$-PABA-1 (Figure $3 \mathrm{C}$ ) and $\mathrm{FG}_{18}-\mathrm{PABA}-3$ carboxylic acid of $\mathrm{FG}_{18}-\mathrm{PABA}-3$ is more than $\mathrm{FG}_{18^{-}}$ (Figure 3E). However, in turn, the peak's width of PABA-1.

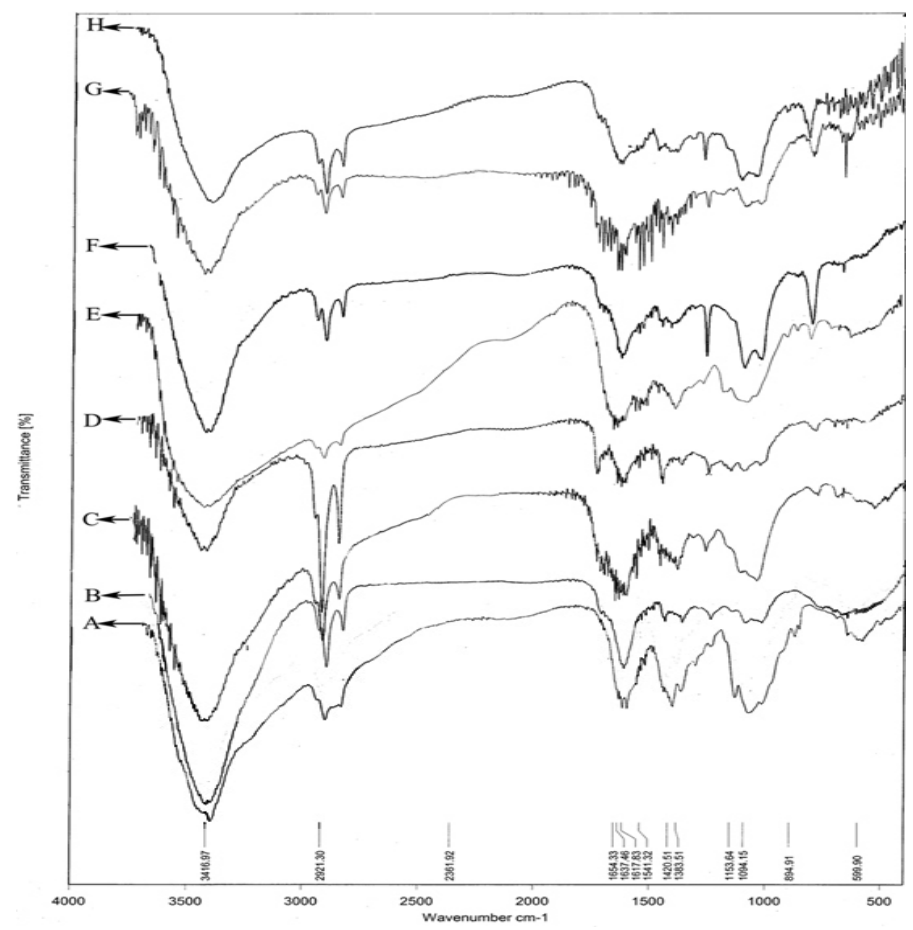

Figure 4. Michael addition reaction of acrylic acid with chitosan.
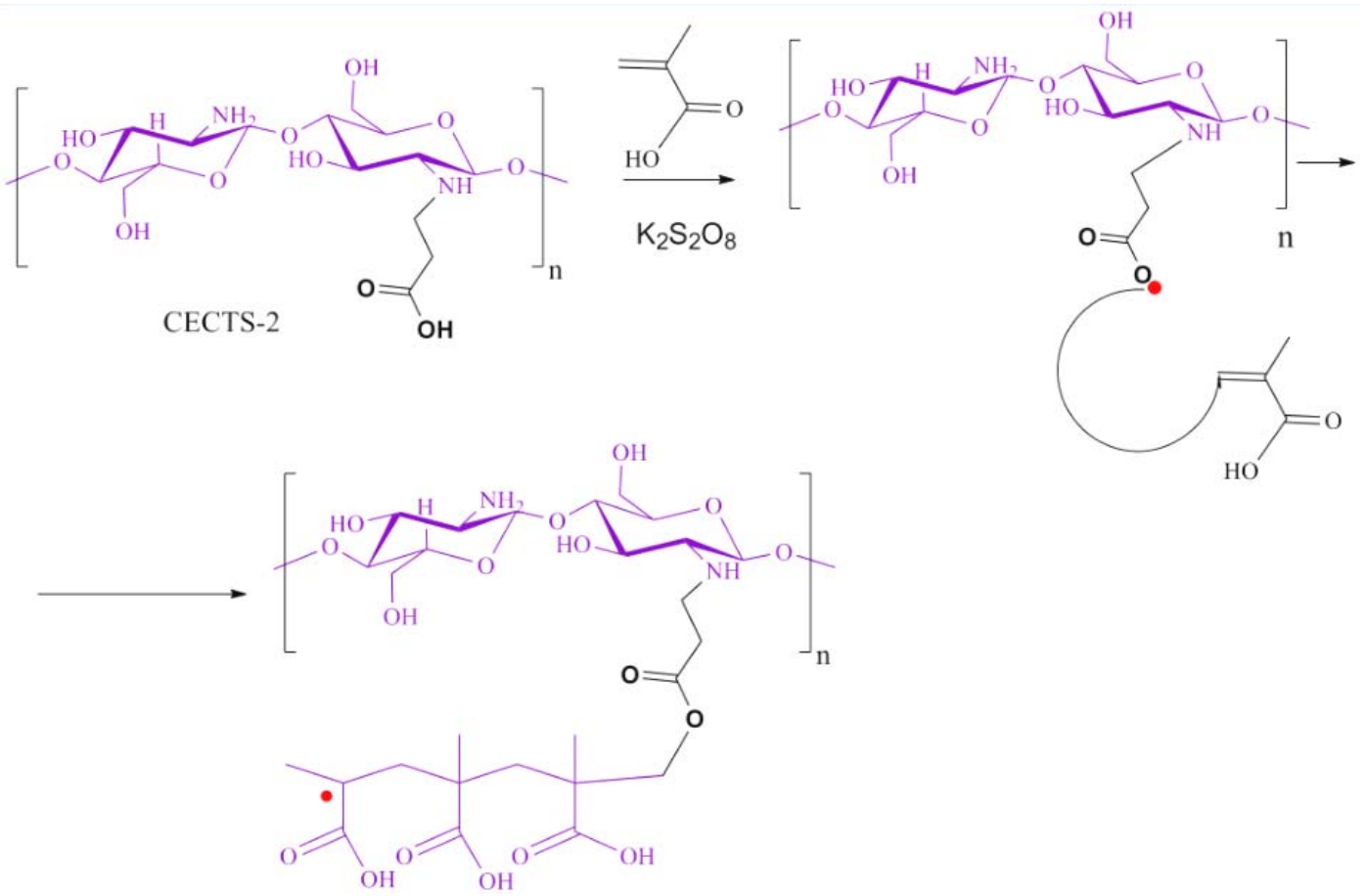

Figure 5. Graf copolymerization to synthesis CECTS-2-g-PMMA. 
Synthesis of CECTS-1 and CECTS-2: As could be seen in figure 4, water-soluble CECTS-1 and CECTS-2 were successfully synthesized using only water as medium.

This simple procedure will be appropriate as an ecofriendly mild chemical modification process. It has been confirmed that mono-alkylation to the amino groups of the chitosan occur under the mild condition (Aoi et al., 2000). The selection of organic acid as inversing agent of swelling behavior is remarkable in two aspects. It could be used both the proton donor to dissolve chitosan and the reagent for the Michael reaction.

Characterization of CECTS-1 and CECTS-2: CECTS1 and CECTS- 2 were synthesized by the Michael addition reaction of acrylic acid with chitosan, as shown in figure 4. The FT-IR spectrum of CECTS-1, for example, showed a strong and wide band at $3442 \mathrm{~cm}^{-1}$ was the respective absorption of hydroxyl, amine and acid groups. Furthermore, C-H asymmetrical stretching, C-H bending, $\mathrm{N}-\mathrm{H}$ bending and $\mathrm{C}-\mathrm{N}$ stretching vibrations of the CECTS1 could be seen at 2852-2961, 1558, 1558 and $1262 \mathrm{~cm}^{-1}$, respectively. Moreover, the respective peak at $1716 \mathrm{~cm}^{-1}$ could be assigned to the stretching vibration of carbonyl groups of carboxylic acid. The band around 1023 and $1097 \mathrm{~cm}^{-1}$ could be assigned as characteristic stretching vibration of $\mathrm{C}-\mathrm{O}$ of chitosan.

Study of graf copolymerization condition on the synthesis of CECTS-2-g-PMMA: CECTS-2-g-PMMA was synthesized by the graft copolymerization of methacrylic acid as shown in figure 5. The chemical modification was exerted homogeneously in aqueous medium owing to the application of CECTS-2 rather than chitosan, which renders the mixture of MMA and CECTS miscible. Based on our knowledge, the temperatures between $50-80^{\circ} \mathrm{C}$ has the best performance in respect of both Grafting percentage (GP) and Grafting efficiency (GE) (Kang et al., 2006; Sashiwa et al., 2003). In one hand, the reaction between the amino group of CECTS-2 and potassium persulfate (PPS) did not progress easily when the reaction temperature is lower than $50^{\circ} \mathrm{C}$. On the other hand, a higher temperature than $50^{\circ} \mathrm{C}$ was effective in enhancing the possibility of successful collision for PPS and CECTS2 , which resulted in increasing of CECTS-2 macroradicals and consequently enhanced the graft copolymerization of MMA onto CECTS-2. However, GP and GE decreased with a further increase in temperature more than $80^{\circ} \mathrm{C}$, owing to the greater possibilities of termination and chain transfer at a relatively higher reaction temperature (Sun et al., 2003). Thus, we selected the $80^{\circ} \mathrm{C}$ as the optimum temperature for starting graft copolymerizatin. With respect to the solubility of CECTS-2 in aqueous media, we employed the $\mathrm{N}, \mathrm{N}^{\prime}$-methylenebisacrylamide as crosslinking agent in order to decrease the solubility of CECTS2 as well as increase the swelling capacity.

Characterization of CECTS-2-g-PMMA: As shown in figure $3 \mathrm{H}$, strong and wide band at $3426 \mathrm{~cm}^{-1}$ was the absorption of hydroxyl, amine and acid groups of this compound. The peaks at $1716 \mathrm{~cm}^{-1}$ and $1652 \mathrm{~cm}^{-1}$ could be assigned to the stretching vibrations of carbonyl of carboxylic acid and amide groups, respectively. The FT-IR spectrum of this polymer indicated an N-H bending vibration at $1557 \mathrm{~cm}^{-1}$ and stretching vibration of $\mathrm{C}-\mathrm{N}$ at $1262 \mathrm{~cm}^{-1}$. The spectum of the synthesized polymer also depicted a band around 1028 and $1098 \mathrm{~cm}^{-1}$ that could be assigned as characteristic stretching vibration of $\mathrm{C}-\mathrm{O}$. The ratio of peak intensity at 1716 to $1557 \mathrm{~cm}^{-1}$ (Figure $3 \mathrm{H}$ and G) increased with an increase of graft copolymerization, indicating the graft copolymerization of MMA onto CECTS-2.

Swelling of polymers: With study the swelling behavior of PABA-conjugated $F_{6}$ and $F_{18}$, PABAconjugated $\mathrm{FG}_{6}$ did not show inverse swelling behavior. It could be as a result of the lack of enough free aldehyde groups of the glutaraldehyd on the backbone of $\mathrm{FG}_{6}$. So, the $\mathrm{FG}_{18}$ was selected as the suitable option to inverse the swelling behavior. As the next step, we continued the optimization with changing the organic solvent to water as green solvent as well as to increase the inverse swelling behavior. Overall, the change of solvent leads to inversing swelling behavior of PABA-conjugated $\mathrm{FG}_{18}$.

On the other hand, with comparing of $\mathrm{FG}_{18}-\mathrm{PABA}-2$ and $\mathrm{FG}_{18}-\mathrm{PABA}-3$, polymers swelling shows that in $\mathrm{FG}_{18^{-}}$ PABA-2, PABA couldn't go through polymer scaffold due to rigidity and stiffness of network, so it reacts with glutaraldehyde's free ends. By heating, in $\mathrm{FG}_{18}$-PABA-3, PABA acquires enough energy to go through polymer network to inverse swelling behavior. Time-dependent 
swelling behavior of cross-linked polymers at $\mathrm{pH} 1$ and $\mathrm{pH} 7.4$ at $37^{\circ} \mathrm{C}$ was plotted in figure 6 .

Investigation on swelling CECTS- 1 and CECTS-2 at two $\mathrm{pH} 1$ and 7.4 have shown that they have the most swelling at $\mathrm{pH} 1$ as it was almost trepanned to dissolve polymers. As a result, we couldn't inverse chitosan swelling behavior by the Michael addition reaction of acrylic acid but grafting methacrylic acid on the CECTS-2 inversed chitosan swelling behavior as shown in the figure 6 E. Swelling at $\mathrm{pH} 7.4$ is more than $\mathrm{pH} 1$. It could be as a result of presence of carboxylic groups of PMMA in CECTS-2-g-PMMA that not only neutralize the amine groups of chitosan but also by ionization at $\mathrm{pH} 7.4$ lead to electrostatic repulsion in hydrogel and accordingly swelling.

$\mathrm{B}$

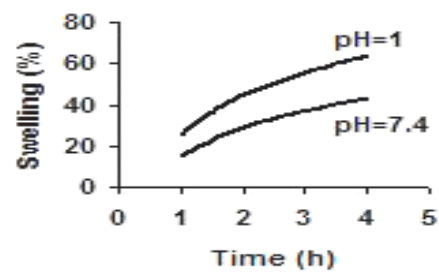

C
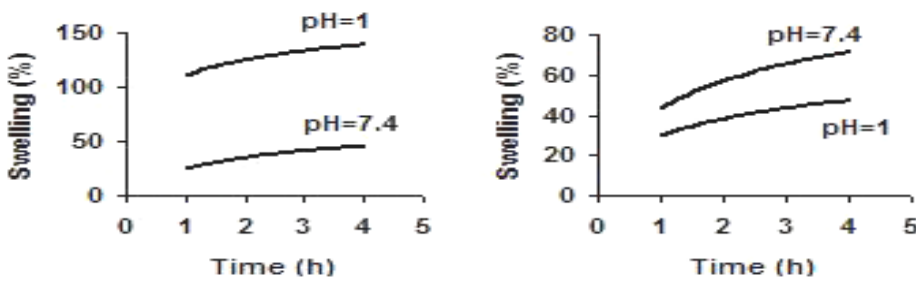

$\mathrm{E}$

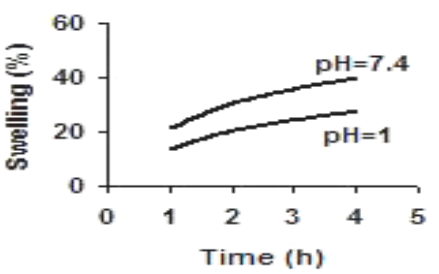

D

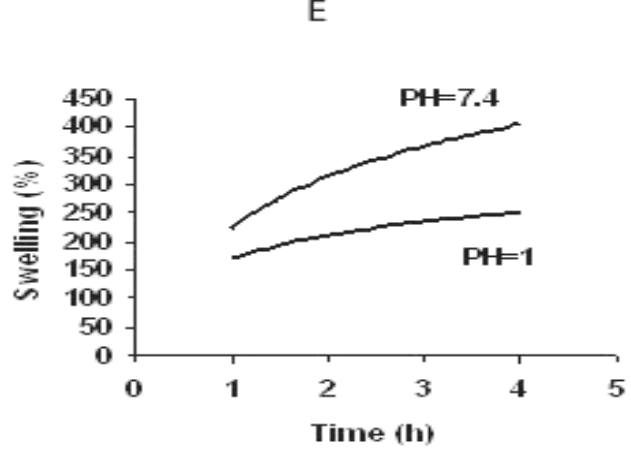

Figure 6. Swelling of polymers of A: FG6-PABA, B: FG18-PABA-1, C: FG18-PABA-2, D: FG18-PABA-3 and E: CECTS-2-g-PMMA.

\section{Conclusions}

With comparison on the swelling behavior of PABAconjugated $\mathrm{FG}_{6}$ and $\mathrm{FG}_{18}$, the $\mathrm{PABA}$-conjugated $\mathrm{FG}_{18}$ at $60^{\circ} \mathrm{C}$ indicated that it could inverse the swelling behavior. In addition, the result of inversing in aqueous solvent is better than that of methanol. Investigation on CECTS-1 and CECTS-2 polymers generated from Michael addition reaction depicted that the most swelling was taken place at $\mathrm{pH} 1.0$ so that it could almost dissolve polymers. As a result, we couldn't inverse chitosan swelling behavior by the Michael addition reaction of AA. However, the graft copolymerization of MMA on the CECTS-2 was successful. In general, the third method among the abovementioned modifications, possessed the best results.

In an effort to obtain self-assembled systems with ability in this work, new types of potentially amphiphilic graft copolymers were synthesized based on hydrophobic chitosan and hydrophilic segments containing polar carboxylic acid groups such as PABA, AA and MMA, respectively. Our experiments to test their amphiphilic 
behavior as potential utilities in biomedical applications are ongoing.

\section{Acknowledgments}

The authors express their appreciations to Chemistry Department Azarbaijan Shahid Madani University and Research Institute for Fundamental Sciences (RIFS), University of Tabriz for the supports of this project.

\section{References}

Ai, H., Wang, F.R., Xia, Y.Q., Chen, X. and Lei, C. 2012. Antioxidant, antifungal and antiviral activities of chitosan from the larvae of housefly, Musca domestica L. Food Chem. 132, 493-498.

Aoi, K., Seki, T., Okada, M., Sato, H., Mizutani. S.I., Ohtani H. 2000. Synthesis of a novel $N$-selective ester functionalized chitin derivative and water-soluble carboxyethylchitin. Macr. Chem. Phys. 201, 1701-1708.

Baumann, H. and Faust, V. 2001. Concepts for improved regioselective placement of O-sulfo, $\mathrm{N}$-sulfo, $\mathrm{N}$-Acetyl and $\mathrm{N}$-carboxymethyl groups in chitosan derivatives. Carbohydr. Res. 331, 43-57.

Benhabiles, M.S., Salah, R., Lounici, H., Drouiche, N., Goosen, M.F.A. and Mameri, N. 2012. Antibacterial activity of chitin, chitosan and its oligomers prepared from shrimp shell waste. Food Hydro. 29, 48-56.

Domingues, R.C.C., Faria, Junior, S.B., Silva, R.B., Cardoso, V.L. and Reis, M.H.M. 2012. Clarification of passion fruit juice with chitosan: Effects of coagulation process variables and comparison with centrifugation and enzymatic treatments. Process Biochem. 47, 467-471.

Fang, M., Long, J., Zhao, W., Wang, L. and Chen, G. 2010. pHResponsive chitosan-mediated graphene dispersions. Langmuir. 26, 16771-16774.

Gerasimenko, D.V., Avdienko, I.D., Bannikova, G.E., Zueva, O. and Varlamov, V.P. 2004. Antibacterial effects of watersoluble low-molecular-weight chitosans on different microorganisms. Prikl Biokhim Mikrobiol. 40, 301-306.

Goosen, M.F.A. (Ed.). 1996. Applications of Chitan and Chitosan. CRC Press.
Jayakumar, R., Prabaharan, M., Nair, S.V. and Tamura, H. 2010. Biomaterials based on chitin and chitosan nanofibers in wound dressing applications. Biotechnol Adv. 28, 142-150.

Kang, H.M., Cai, Y.L. and Liu, P.S. 2006. Synthesis, characterization and thermal sensitivity of chitosan-based graft copolymers. Carbohydr Res. 341, 2851-2857.

Lin, H.R., Chen, Y.C., Lin, Y.J., Ling, M.H., Chou, C.W., Hseu, Y.C. 2014. pH-Sensitive Hollow Alginate-Chitosan Hydrogel Beads for Bitter Gourd Delivery. Int. J. Polym. Mate.r Polym. Biomater. 63, 41-47.

Meng, Q., Hu, J., Ho, K., Ji, F. and Chen, S. 2009. The Shape Memory Properties of Biodegradable Chitosan/Poly(1lactide) Composites. J. Polym. Environ. 17, 212-224.

Park, J.H., Cho, Y.W., Chung, H., Kwon, I.C. and Jeong, S.Y. 2003. Synthesis and characterization of sugar-bearing chitosan derivatives: aqueous solubility and biodegradability. Biomacromolecules 4, 1087-1091.

Saber-Samandari, S., Yilmaz, O. and Yilmaz, E. 2012. Photoinduced Graft Copolymerization Onto Chitosan Under Heterogeneous Conditions. J. Macromol. Sci. A. 49, 591598.

Saita, K., Nagaoka, S., Shirosaki, T., Horikawa, M., Matsuda, S. and Ihara, H. 2012. Preparation and characterization of dispersible chitosan particles with borate crosslinking and their antimicrobial and antifungal activity. Carbohydr Res. 349, 52-58.

Sashiwa, H., Yamamori, N., Ichinose, Y., Sunamoto, J. and Aiba, S.I. 2003. Chemical Modification of Chitosan, 17a Michael Reaction of Chitosan with Acrylic Acid in Water. Macromol. Biosci. 3, 231-233.

Strand, S.P., Tommeraas, K., Varum, K.M. and Ostgaard, K. 2001. Electrophoretic light scattering studies of chitosans with different degrees of $\mathrm{N}$-acetylation. Biomacromolecules 2, 1310-1314.

Sun, T., Xu, P., Liu, Q., Xue, J. and Xie, W. 2003. Graft copolymerization of methacrylic acid onto carboxymethyl chitosan. Eur. Polym. J. 39, 189-192.

Wang, J. and Wang, H. 2011. Preparation of soluble paminobenzoyl chitosan ester by Schiff's base and antibacterial activity of the derivatives. Int. J. Biol. Macromol. 48, 523-529. 